\title{
A review on polyurethane and its composites
}

\begin{abstract}
In the recent years, the research about polyurethane (PU) composites (thermoplastic, thermoset, biobased polyurethane with synthetic fibers (glass, aramid and carbon) and natural fibers used as reinforcement of polymers has been increased due to their biodegradability, lightness, reduced cost and favorable mechanical properties. Unique mechanical, thermal, and chemical properties of Polyurethanes (thermoset/thermoplastic) can be designed by the reaction of various polyhydric compounds (polyols) and polyisocyanates which is derived from the formation of cross-linked polyurethanes. One of the challenges that researchers face today is to achieve satisfactory interfacial bonding which will result in products with better mechanical and thermal properties. Composites having better mechanical and thermal properties could find more industrial applications and consequently would have greater commercial acceptance. However, this is difficult due to the hydrophilicity of the fibers and the hydrophobicity of polymers such as polyurethane. In this review paper, comprehensive review about PU and its polymer composites were presented with concentrating on the effect of the different kinds of natural and synthetic fibers on the PU based polymer composites products. We also discussed the effect of chemical treatments of natural fibers on improvement of interfacial bonding between natural fiber and polyurethane matrix for development of advanced materials with better mechanical and thermal properties.
\end{abstract}

Keyword: Polyurethane; Thermoset polyurethane; Thermoplastic polyurethane; Polyurethane composites 